\title{
Perfil da alimentação oferecida em instituições geriátricas do Distrito Federal
}

\author{
Profile of the food offered in the elderly homes \\ of Distrito Federal
}

Natacha TORAL ${ }^{1}$

Muriel Bauermann GUBERT²

Bethsáida de Abreu Soares SCHMITZ2,3

\section{R E S U M O}

\section{Objetivo}

Avaliar o padrão da alimentação oferecida, o setor de produção de refeições e a inclusão de profissionais de saúde em instituições geriátricas do Distrito Federal.

\section{Métodos}

Trata-se de um estudo descritivo, transversal, realizado em cinco instituições geriátricas, incluindo 244 idosos, o que corresponde a 25,0\% dos idosos institucionalizados no Distrito Federal em 2002. O setor de produção de refeições foi avaliado pelas condições higiênicas e ambientais/estruturais. Investigou-se a inclusão de profissionais de saúde no quadro de funcionários das instituições, o número de refeições oferecidas por dia aos idosos, as opções de consistência da alimentação e a presença de um cardápio semanal impresso. Foi realizada uma avaliação qualitativa da adequação nutricional da alimentação oferecida em comparação com as recomendações da Pirâmide Alimentar.

\section{Resultados}

Observaram-se condições higiênicas e ambientais/estruturais precárias, além da escassez de profissionais de saúde em todas as instituições. A maioria destas oferecia cinco refeições diárias, com opções de consistência normal e semilíquida. Apenas duas instituições apresentavam cardápios semanais impressos. A avaliação nutricional qualitativa mostrou que os grupos de alimentos com maior inadequação na oferta foram: hortaliças, com oferta média de apenas 53,0\% da recomendação, e frutas, com 60,0\% da recomendação. A oferta de alimentos do grupo dos óleos e gorduras foi considerada excessiva (120,0\% da recomendação).

\section{Conclusão}

A abundância de fatores inadequados no funcionamento do setor de produção de refeições, a falta de profissionais da saúde e o desequilíbrio entre grupos alimentares oferecidos apontam para um quadro preocupante quanto à qualidade de vida dos idosos institucionalizados do Distrito Federal.

Termos de Indexação: alimentação institucional; asilos para idosos; avaliação nutricional; idoso.

\footnotetext{
1 Mestranda, Faculdade de Saúde Pública, Universidade de São Paulo. Av. Dr. Arnaldo, 715, 01246-904, São Paulo, SP, Brasil. Correspondência para/Correspondence to: N. TORAL.E-mail: <natytb@usp.br>.

2 Observatório de Políticas de Segurança Alimentar e Nutrição, Núcleo de Pesquisas em Políticas Públicas, Universidade de Brasília. Brasília, DF, Brasil.

${ }^{3}$ Departamento de Nutrição, Universidade de Brasília. Brasília, DF, Brasil.
} 


\section{A B S T R A C T}

\section{Objective}

To evaluate institutional feeding, meal production facilities and the inclusion of healthcare professionals in elderly homes of Distrito Federal.

\section{Methods}

This is a descriptive and cross-sectional study that was carried out in five elderly homes. Two hundred fortyfour seniors were included, corresponding to 25\% of the institutionalized seniors in Distrito Federal in 2002. The meal production facility was evaluated based on its hygienic and environmental-structural conditions. The inclusion of healthcare professionals among the staff of the elderly homes, the number of meals offered daily, the meal consistency options and the presence of a printed weekly menu were investigated. A qualitative evaluation of the nutritional adequacy of the institutional feeding was done based on the Food Guide Pyramid recommendations.

\section{Results}

Precarious hygienic and environmental-structural conditions were identified. Healthcare professionals were scarce in all homes. Most homes offered five daily meals with two consistency options: normal and semiliquid. Only two homes had a printed weekly menu. The nutritional evaluation of meals showed that the most inadequate percentages of food groups offered were vegetables, with a median offer of only $53 \%$ of the recommendation, followed by fruits, with $60 \%$ of the recommendation. The offered percentage of the oil and fat group was considered excessive (120\% of the recommendation).

\section{Conclusion}

Abundant inadequate factors in meal production facilities, the poor inclusion of healthcare professionals and the imbalance in the food groups offered point to a worrying situation for the quality of life of institutionalized seniors in Distrito Federal.

Indexing terms: institutional feeding; homes for the aged; nutrition assessment; aged.

\section{N T R O D U Ç Ã O}

A redução nas taxas de mortalidade e natalidade ocorrida nas últimas décadas no Brasil, como parte do fenômeno da transição demográfica, ocasionou alterações significativas na pirâmide populacional. O envelhecimento dos brasileiros tem despertado o interesse das políticas de saúde pública, tendo em vista a demanda social implicada nesse fenômeno, já observado em países desenvolvidos ${ }^{1-3}$. Atualmente, os problemas do sistema público de saúde, do sistema previdenciário e das condições de aposentadoria geram um processo deficiente de inclusão social do idoso no País ${ }^{4,5}$. O avançar da idade propicia o estabelecimento de doenças incapacitantes, levando à dependência de cuidados externos, sejam eles provenientes da própria família ou de profissionais especializados no atendimento geriátrico, o que aumenta a demanda por instituições de abrigo ou asilos para atender às necessidades dessa população ${ }^{1,6}$.
Os aspectos relativos à alimentação oferecida pelos asilos são essenciais, considerando o grande impacto dos hábitos alimentares do idoso no seu estado de saúde ${ }^{7}$. A Portaria n 810 , do Ministério da Saúde, sancionada em 1989, estabelece as normas para o funcionamento de instituições geriátricas e destaca a responsabilidade da própria instituição no que diz respeito à produção de refeições para os internos ${ }^{8}$. Dessa forma, o planejamento adequado da alimentação, considerando as características nutricionais e higiênico-sanitárias, cabe aos responsáveis pelo setor de produção de refeições do asilo ${ }^{6}$.

O último censo demográfico realizado no Brasil identificou que 5,3\% da população do Distrito Federal, ou seja, 109.638 habitantes apresentavam idade igual ou superior a 60 anos, o que corresponde ao critério de definição de indivíduo idoso 9,10 . Destes, aproximadamente 10,0\% encontram-se institucionalizados nos 16 asilos e abrigos geriátricos existentes no Distrito 
Federal de acordo com informações fornecidas em 2002, pelo Conselho do Idoso da Secretaria de Trabalho e Direitos Humanos do governo local.

Tendo em vista a escassez de trabalhos sobre o tema e a relevância da associação entre a alimentação e a saúde do idoso, este estudo teve como objetivo avaliar o setor de produção de refeições, comparar o padrão da alimentação oferecida com as recomendações nutricionais, e identificar a composição da equipe de profissionais de saúde de instituições geriátricas do Distrito Federal.

\section{MÉ T O D O S}

Este trabalho trata de um estudo descritivo e transversal, realizado nos meses de agosto e setembro de 2002. Para definição da população de abrangência e cálculo da amostra, utilizaram-se informações oficiais do Conselho do Idoso da Secretaria de Trabalho e Direitos Humanos do Governo do Distrito Federal. Constatou-se que havia no Distrito Federal 16 instituições de abrigo de idosos em funcionamento. Dessas, apenas oito instituições preenchiam os critérios de inclusão adotados neste estudo: estarem localizadas em centros urbanos próximos ao Plano Piloto e não se caracterizarem por clínicas geriátricas. Todas correspondiam a instituições de assistência social, cujos recursos financeiros eram provenientes principalmente de doações.

As instituições selecionadas foram contatadas para avaliação do interesse em participar da pesquisa. Os procedimentos foram apresentados aos responsáveis pelos locais, garantindo a não-identificação das instituições para fins de publicação, caso fosse informado o consentimento. Houve recusa de participação de três instituições, finalizando a amostra em cinco instituições. Estas abrangiam um total de 244 idosos, representando, aproximadamente, 25,0\% dos idosos institucionalizados no Distrito Federal em 2002, e 0,2\% da população geriátrica dessa região?
Foram coletados dados relativos às condições de funcionamento e à alimentação oferecida aos idosos, no intuito de: avaliar as condições higiênicas e ambientais do local de produção de alimentos, caracterizar qualitativamente a oferta de alimentos à população institucionalizada, verificar a presença de profissionais de saúde atuando nessas instituições.

Por meio de um check-list elaborado para este estudo, avaliaram-se as condições higiênicas e as condições estruturais e ambientais do setor de produção de refeições, tendo por base as diretrizes da Resolução n³3/77, da Comissão Nacional de Normas e Padrões para Alimentos ${ }^{11}$ e a Portaria n¹428/93 do Ministério da Saúde ${ }^{12}$. Foram observadas características como a planta do estabelecimento, materiais de revestimento, instalações elétricas e hidráulicas, serviços básicos de saneamento, equipamentos, utensílios, condições de armazenagem e fluxo da produção. A avaliação classificou o setor de produção como adequado quando, no mínimo, 75\% dos itens observados estavam de acordo com as referidas normas, tanto para as condições higiênicas, como para as condições estruturais e ambientais. Caso contrário, o setor de produção foi considerado inadequado.

Para avaliação da alimentação fornecida, investigaram-se, mediante entrevista com os responsáveis pelas instituições, o número de refeições oferecidas por dia aos idosos, as opções de consistência da alimentação e a presença de um cardápio semanal impresso. Para a análise de consistência, considerou-se como dieta normal aquela sem nenhuma modificação dietoterápica com a finalidade de facilitar a digestão ou aumentar sua tolerância; dieta pastosa foi considerada aquela com alterações na consistência, de forma a torná-la de maior digestibilidade e de menor consistência em relação à dieta normal; dieta semilíquida foi considerada aquela composta por líquidos e por alimentos semi-sólidos, a qual requer um mínimo de processo digestivo.

Para o cálculo de adequação nutricional, foram registradas todas as preparações oferecidas 
aos idosos no dia anterior à entrevista. A partir desse registro, foi realizada uma avaliação qualitativa de sua adequação nutricional em comparação com as recomendações propostas pela Pirâmide Alimentar adaptada por Philippi et al. ${ }^{13}$. Tendo em vista que o foco do estudo não foi quantitativo, foi considerado o oferecimento de uma porção para cada uma das preparações do cardápio, de modo a possibilitar o cálculo do número de porções oferecidas de cada grupo alimentar: pães, cereais, raízes e tubérculos; hortaliças; frutas; carnes e ovos; leite e derivados; leguminosas; óleos e gorduras; açúcares e doces. O valor obtido foi comparado ao valor médio da faixa de consumo recomendado para cada grupo, sendo calculado o percentual de adequação da oferta relativo a essa recomendação. Para facilitar a comparação visual com a Pirâmide Alimentar, foi desenvolvida uma pirâmide, em moldes semelhantes aos da referência, a partir da distribuição média dos grupos alimentares observadas nas instituições.

Investigou-se também a presença de qualquer profissional de saúde nas instituições geriátricas. Tal identificação referiu-se apenas à inclusão desses profissionais no quadro de funcionários do local. Não foi considerada, portanto, atuação voluntária ou caracterizada por consultoria ocasional desses profissionais.

A tabulação e a análise dos dados coletados foram realizadas com auxílio do programa de computador Epi Info versão 6.04 ${ }^{14}$.

\section{RESULTA DOS}

Todos os setores de produção avaliados apresentaram inadequação nas condições relativas a estrutura, ambiente e higiene (Figura 1). Em relação às condições estruturais e ambientais, nenhuma das cinco instituições avaliadas atendeu ao mínimo de 75,0\% de adequação. As condições de higiene variaram entre $0,0 \%$ e $75,0 \%$, sendo o valor máximo alcançado por apenas uma instituição.

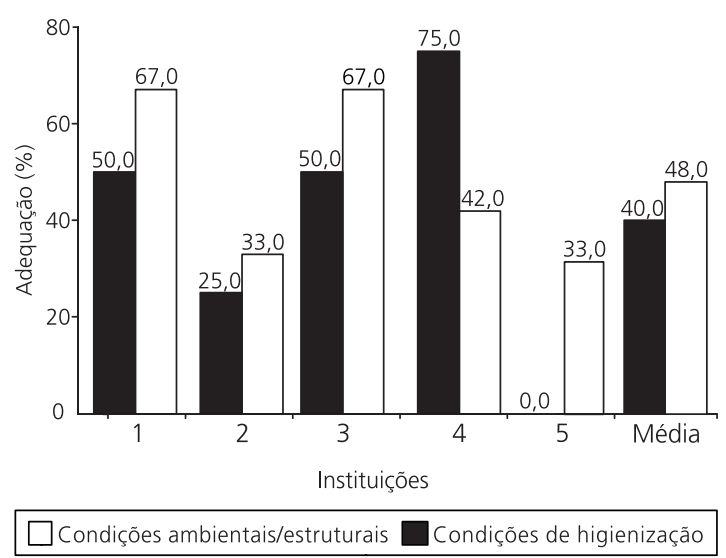

Figura 1. Distribuição da adequação das condições ambientais /estruturais e de higienização do setor de produção de refeições das instituições geriátricas. Distrito Federal, 2002

Três instituições $(60,0 \%)$ ofereciam cinco refeições diárias aos idosos. Somente uma instituição oferecia seis refeições, enquanto outro local oferecia quatro refeições ao dia.

Todas as instituições forneciam refeições com consistência normal, para a maioria dos idosos, e semilíquida, para aqueles que apresentavam dificuldade em se alimentar. Duas instituições $(40,0 \%)$ apresentavam também refeições com consistência pastosa.

Apenas duas instituições apresentavam cardápios semanais impressos, sendo que um destes continha apenas as refeições principais. Cabe destacar que, na ausência de nutricionista nas instituições visitadas, os mesmos haviam sido elaborados pelo assistente social e pelo superintendente de cada local.

A avaliação nutricional qualitativa dos cardápios registrados mostrou que os grupos de alimentos com maior inadequação na oferta, em comparação com as recomendações, foram o das hortaliças, com oferta média de apenas 53,0\% para cada, seguido das frutas $(60,0 \%)$, enquanto a oferta de leguminosas e de alimentos do grupo dos óleos e gorduras foi considerada excessiva (120,0\% da recomendação) (Tabela 1). A comparação visual entre o preconizado pela Pirâmide Alimentar adaptada e a distribuição da oferta dos grupos alimentares observada nas instituições é apresentada na Figura 2. 
Tabela 1. Distribuição percentual da adequação entre a oferta dos grupos alimentares observada no cardápio das instituições geriátricas e as recomendações da Pirâmide Alimentar. Distrito Federal, 2002.

\begin{tabular}{lccr}
\hline $\begin{array}{l}\text { Grupos de } \\
\text { alimentos }\end{array}$ & $\begin{array}{c}\text { Número médio } \\
\text { de porções } \\
\text { oferecidas/dia }\end{array}$ & $\begin{array}{c}\text { Número médio } \\
\text { de porções } \\
\text { recomendadas/ } \\
\text { dia* }\end{array}$ & $\begin{array}{c}\text { Adequação } \\
(\%)\end{array}$ \\
\hline Pães e massas & 6,4 & 7,0 & 91,4 \\
Hortaliças & 2,4 & 4,5 & 53,3 \\
Frutas & 2,4 & 4,0 & 60,0 \\
Leite e derivados & 3,0 & 3,0 & 100,0 \\
Leguminosas & 1,2 & 1,0 & 120,0 \\
Carnes e ovos & 1,6 & 1,5 & 106,7 \\
Açúcares e doces & 0,8 & 1,5 & 53,3 \\
Oleos e gorduras & 1,8 & 1,5 & 120,0 \\
\hline
\end{tabular}

* Valores médios nas faixas de consumo recomendadas pela Pirâmide Alimentar ${ }^{12}$.

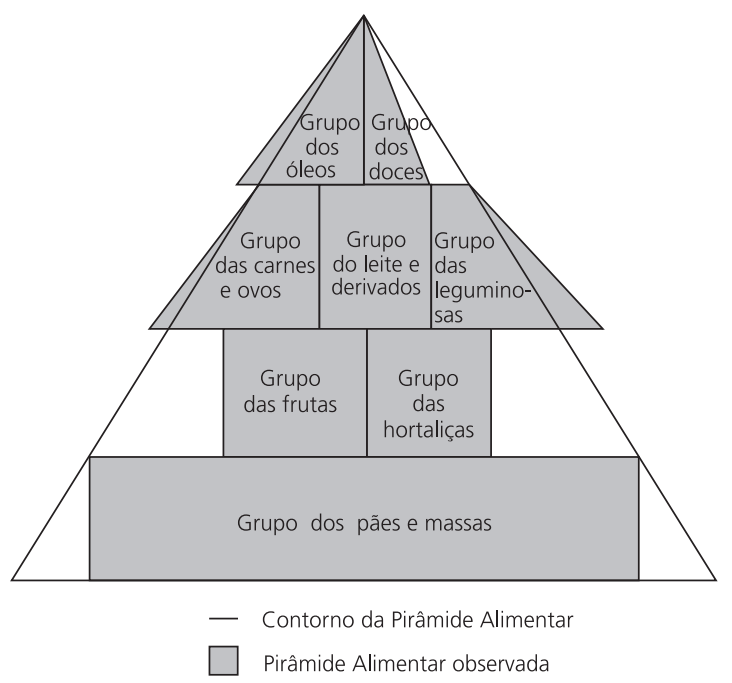

Figura 2. Comparação proporcional entre as recomendações da Pirâmide Alimentar e a oferta dos grupos alimentares observada no cardápio das instituições geriátricas. Distrito Federal, 2002.

Quatro instituições dispunham de técnicos em enfermagem, entretanto a atuação de enfermeiros foi identificada em apenas duas instituições. Nenhum médico, nutricionista, fisioterapeuta, psicólogo ou outro profissional de saúde fazia parte do quadro de funcionários fixos das instituições geriátricas.

\section{I S C U S S Ã O}

Não existem dados oficiais quanto ao número de idosos institucionalizados no Brasil.
Apenas um estudo, datado de 1984, citado por Born ${ }^{15}$, estimou que entre $0,6 \%$ e 1,3\% da população idosa se encontrava em instituições. Chaimovicz ${ }^{1}$ identificou uma prevalência de 0,8\% de institucionalização entre os idosos da região metropolitana de Belo Horizonte em 1992, valor muito inferior ao encontrado neste estudo. Segundo informações do Conselho do Idoso da Secretaria de Trabalho e Direitos Humanos do governo local, cerca de $10,0 \%$ dos idosos do Distrito Federal encontram-se institucionalizados, o que pode ser atribuído ao aumento crescente da população nesse estágio de vida no Brasil e no mundo.

Este trabalho identificou a ausência de nutricionista em todas as instituições avaliadas. Resultados semelhantes foram encontrados por diversos autores ${ }^{6,16,17}$, o que representa um maior risco de inadequação nutricional da alimentação dos idosos. Marucci ${ }^{16}$ observou que nenhuma das instituições geriátricas estudadas oferecia quantidade adequada em termos de energia e nutrientes. O delineamento deste estudo não permitiu uma análise quantitativa da alimentação, mas a avaliação qualitativa mostrou inadequações. Acredita-se que a fraca, ou mesmo ausente atuação de nutricionistas nesses locais seja decorrente da dificuldade financeira encontrada na maioria das instituições, frente aos recursos elevados que demanda a composição do quadro de funcionários ${ }^{6,15}$.

A verificação da presença de nutricionistas atuando em asilos é de extrema importância, tendo em vista que este profissional é capacitado para o cálculo da adequação nutricional das preparações que serão oferecidas aos idosos, considerando as alterações fisiológicas e as disfunções degenerativas características desse estágio de vida ${ }^{15}$. Além disso, o nutricionista é encarregado da elaboração de cardápios e da coordenação do setor de produção de refeições, de forma a garantir a manutenção da saúde, a prevenção de doenças ou a recuperação destas por meio de uma alimentação saudável ${ }^{18}$. 
Os setores de produção das instituições geriátricas visitadas apresentavam condições ambientais, estruturais e higiênicas extremamente deficientes. A maioria apresentou classificação insatisfatória para os fatores avaliados, o que constitui um risco acentuado para ocorrência de doenças veiculadas pelos alimentos. Essa situação é favorecida pela "fragilidade" do idoso, correspondente a um estado de debilidade do sistema imunológico, resultando numa susceptibilidade maior a doenças'.

Viana ${ }^{6}$ avaliou as unidades de alimentação e nutrição de 117 instituições geriátricas de São Paulo e verificou que a maioria atendia às recomendações quanto à estrutura física, operacional e organizacional. Contudo, foram identificados aspectos falhos, como o desconhecimento da legislação sanitária e dos riscos envolvidos na produção de alimentos, bem como o baixo nível de escolaridade e qualificação dos funcionários envolvidos e atuação pouco significativa ou mesmo inexistente de nutricionistas nas unidades.

As instituições visitadas no presente estudo ofereciam, no mínimo, quatro refeições diárias, resultado semelhante ao observado por Marucci ${ }^{19}$, que identificou que $85 \%$ dos 308 idosos matriculados em ambulatório geriátrico realizavam quatro ou mais refeições ao dia. Viana ${ }^{6}$ observou que $69,2 \%$ das instituições geriátricas estudadas ofereciam cinco refeições diárias. Tais resultados são satisfatórios, uma vez que o fracionamento das refeições é recomendado para evitar longos períodos de jejum, mantendo níveis glicêmicos adequados no sangue para um suprimento energético celular eficiente. A oferta mais freqüente de refeições de menor volume a essa população também deve ser priorizada, em função das alterações decorrentes do envelhecimento. Entre elas, podem ser citados: problemas dentários, prejuízo da produção de enzimas e sucos gástricos, diminuição da percepção sensorial (visão, sabor e aroma dos alimentos é prejudicada) e do metabolismo basal, que levam a uma redução do apetite e do prazer de comer entre os idosos ${ }^{19,20}$.
O oferecimento de no mínimo duas opções de consistência da alimentação, observado em todas as instituições, é um fato esperado e condizente com o atendimento ao idoso, tendo em vista as alterações na capacidade mastigatória nesses indivíduos. Suas principais causas incluem o aparecimento freqüente de cáries e doenças periodontais, o uso de próteses inadaptadas ou em péssimo estado de conservação e a ausência de dentes ${ }^{21}$. Foi observado que nenhuma instituição utilizava os termos "semilíquida" ou "pastosa", optando pelas expressões leigas como "batida no liqüidificador" e "amassada com o garfo", respectivamente. Essa situação expressa o caráter de informalidade e a baixa qualificação dos funcionários envolvidos na produção de refeições.

A avaliação qualitativa da alimentação oferecida aos idosos foi realizada por meio da comparação com as recomendações nutricionais de adultos, já que as recomendações específicas para a população idosa não se encontram bem estabelecidas na literatura, devido às alterações estruturais e funcionais sempre presentes nesses indivíduos ${ }^{20}$.

Verificou-se um oferecimento deficiente de hortaliças e frutas na alimentação dos idosos institucionalizados, com percentuais de adequação de $53 \%$ e $60 \%$, respectivamente, em comparação com a recomendação média de consumo da Pirâmide Alimentar. Dietas pobres nesses grupos alimentares entre a população idosa, fato também encontrado por Souza \& Moreira ${ }^{2}$ e Marucci ${ }^{19}$, constituem um risco de deficiência de micronutrientes, que correspondem às principais fontes de vitaminas e minerais. Diversos estudos têm mostrado a ingestão inadequada desses nutrientes entre idosos, residentes tanto em asilos como em seus próprios domicílios, seja pelas condições socioeconômicas, presença de doenças, alterações no modo de vida dessa população ou pelo hábito alimentar inadequado ${ }^{21-23}$.

O baixo consumo de hortaliças e frutas sugere que a dieta oferecida é pobre em fibras, o que, associado à consistência semilíquida da 
alimentação e à baixa ingestão de líquidos, pela diminuição da sensibilidade à sede, pode favorecer a ocorrência de constipação nos idosos ${ }^{21}$. Dietas com baixo teor de fibras entre idosos também têm sido relatadas na literatura $19,22,24$.

A oferta dos grupos alimentares fontes de proteína (leite e derivados, leguminosas, carnes e ovos) apresentou adequação igual ou superior a $100,0 \%$, e a oferta do grupo dos óleos e gorduras atingiu $120,0 \%$ de adequação. Falque-Madrid et al. ${ }^{24}$ também observaram a tendência do consumo de dietas ligeiramente hiperprotéicas e hiperlipídicas entre idosos venezuelanos. Tal hábito alimentar exige atenção, pois o excesso desses grupos alimentares deve ser evitado, tanto pelos riscos para a saúde cardiovascular dos idosos, como pela dificuldade de digestão por fatores fisiológicos próprios do envelhecimento ${ }^{19}$.

A baixa oferta do grupo dos açúcares e doces, bem como do grupo dos pães e massas, pode contribuir para um consumo deficiente de energia e carboidratos na dieta dos idosos. O consumo energético insuficiente nessa população, freqüentemente observado na literatura ${ }^{22-24}$, pode prejudicar o estado nutricional do idoso e dificultar o alcance das recomendações de micronutrientes, favorecendo o estabelecimento de carências nutricionais. Contudo, o consumo de açúcares e doces não deve ser estimulado, pois seu uso excessivo na alimentação relaciona-se à maior incidência de cárie dental e alteração dos níveis lipídicos e glicêmicos.

Os cardápios implementados nas instituições haviam sido elaborados pelo superintendente do local ou pelo assistente social. Porém, segundo resolução do Conselho Federal de Nutricionistas, é atribuição específica do nutricionista o planejamento de cardápios de acordo com as necessidades de sua clientela, considerando o embasamento científico desse profissional para contemplar as alterações fisiológicas relacionadas ao envelhecimento, como citado anteriormente ${ }^{16}$.

A ausência de um nutricionista nas instituições pode favorecer a presença de condições inadequadas, como as observadas nos setores de produção de refeições dos locais visitados: cruzamento entre fluxos dentro da área de produção, separação deficiente de despensa de alimentos perecíveis e semiperecíveis, utilização comum de área para recebimento de alimento in natura, preparo final das refeições e armazenamento inadequado de alimentos, entre outros fatores que comprometem a qualidade higiênico-sanitária da alimentação oferecida por esses locais.

No Distrito Federal, foi verificada a escassez de profissionais da saúde nas instituições geriátricas. Da mesma forma, em Belo Horizonte, $98 \%$ das instituições não contavam com enfermeiros, 96\% dispunham de atendimento médico apenas voluntário e esporádico e menos de $15 \%$ ofereciam atividades de fisioterapia, terapia ocupacional e psicologia ${ }^{1}$.

Observou-se que é dada preferência aos técnicos em enfermagem em relação aos profissionais com formação acadêmica (enfermeiros), possivelmente devido ao maior custo para a instituição para incluir profissionais de nível superior no quadro de funcionários fixos. Born ${ }^{15}$ ressalta esse problema, freqüentemente observado no Brasil, em associação com a falta de mão-de-obra com o mínimo de qualificação para o atendimento ao idoso.

\section{CONCLUSÃO}

A abundância de fatores inadequados no funcionamento do setor de produção de refeições e o desequilíbrio entre os grupos alimentares oferecidos, bem como a inclusão deficiente de profissionais de saúde nas instituições geriátricas do Distrito Federal apontam para um quadro preocupante quanto à qualidade de vida dos idosos.

Observou-se escassez de estudos tanto de avaliação do setor de produção de refeições desses locais, como do perfil nutricional de idosos institucionalizados no país. São necessárias novas pesquisas que também incluam a avaliação do 
estado nutricional e da alimentação oferecida nesses locais em termos quantitativos, de forma a averiguar o impacto da precária situação observada na saúde desses indivíduos. A avaliação constante e aprofundada da situação do idoso no Brasil está inserida no contexto da valorização deste indivíduo na sociedade e constitui o passo inicial para a implementação de políticas públicas que garantam qualidade de vida ao longo do processo de envelhecimento da população.

\section{A GRADECIMENTOS}

Às nutricionistas Juliana Frossard e Ana Paula Rezende pela colaboração na coleta e análise dos dados deste estudo.

\section{REFER Ê NCIAS}

1. Chaimowicz F. A saúde dos idosos brasileiros às vésperas do século XXI: problemas, projeções e alternativas. Rev Saúde Pública. 1997; 31(2): 184-200

2. Souza FTF, Moreira EAM. Qualidade de vida na terceira idade: saúde e nutrição. Rev Cien Saúde. 1998; 17(2):55-76.

3. Costa MFFL, Guerra HL, Barreto SM, Guimarães RM. Diagnóstico da situação de saúde da população idosa brasileira: um estudo da mortalidade e das internações hospitalares públicas. Informe Epidemiológico do SUS. 2000; 9(1):23-41.

4. Veras RP. Atenção preventiva ao idoso: uma abordagem de saúde coletiva. In: Papaléo Netto M. Gerontologia. São Paulo: Atheneu; 1997. p.383-8.

5. Tavares EL, Anjos LA. Perfil antropométrico da população idosa brasileira. Resultados da Pesquisa Nacional sobre Saúde e Nutrição. Cad Saúde Pública. 1999; 15(4):759-68.

6. Viana IC. Unidades de alimentação e nutrição (UANs) de instituições geriátricas: estrutura física, operacional e organizacional [dissertação]. São Paulo: Faculdade de Saúde Pública, Universidade de São Paulo; 2000.

7. Najas MS, Andreazza R, Souza ALM, Sachs A, Guedes ACB, Sampaio LR, et al. Padrão alimentar de idosos de diferentes estratos socioeconômicos residentes em localidade urbana da região Sudeste, Brasil. Rev Saúde Pública. 1994; 28(3):187-91.
8. Brasil. Ministério da Saúde. Portaria n 810, de 22 de setembro de 1989. Normas para funcionamento de casas de repouso, clínicas e hospitais geriátricos e de outras instituições destinadas ao atendimento de idosos. Diário Oficial da República Federativa do Brasil, Brasília; 27 de setembro de 1989. Seção I, p.17297-8.

9. Brasil. Instituto Brasileiro de Geografia e Estatística [Internet]. Censo demográfico: 2000. Rio de Janeiro; 2000 [acesso 20 abr. 2004]. Disponível em: http://www.ibge.gov.br/censo/default.php

10. World Health Organization. Physical status: the use and interpretation of anthropometry. Genebra; 1995. p.375-407.

11. Brasil. Comissão Nacional de Normas e Padrões para Alimentos. Resolução n 33, de 09 de novembro de 1977. Estabelece os princípios gerais de higiene a serem observados na obtenção, manipulação, armazenagem, transporte e distribuição de alimento, sem prejuízo de normas específicas de higiene a serem estabelecidas para cada espécie de alimento. Diário Oficial da União [Internet]. 1977. Poder Executivo, 9 de novembro. [acesso em15 abr. 2005]. Disponível em: http://elegis.bvs.br/leisref/public/showAct.php?id= $6321 \&$ word =

12. Brasil. Ministério da Saúde. Portaria $n^{\circ} 1428$, de 26 de novembro de 1993. Aprova o Regulamento Técnico para Inspeção Sanitária de Alimentos, as Diretrizes para o Estabelecimento de Boas Práticas de Produção e de Prestação de Serviços na Área de Alimentos e o Regulamento Técnico para o Estabelecimento de Padrão de Identidade e Qualidade (PIQ's) para Serviços e Produtos na Área de Alimentos. Diário Oficial da União [Internet]. 1993; Poder Executivo, 2 de dezembro. [acesso em 28 maio 2004]. Disponível em: http://elegis.bvs.br/leisref/public/showAct.php?id=661

13. Philippi ST, Latterza AR, Cruz ATR, Ribeiro LC. Pirâmide alimentar adaptada: guia para escolha dos alimentos. Rev Nutr. 1999; 12(1):65-80.

14. Center of Disease Control and Prevention. Software: Epi Info Version 6. The Division of Suerveillance and Epidemiology. Epidemiology Program Office. Atlanta, Georgia; 1994.

15. Born T. Cuidado ao idoso em instituição. In: Papaléo Netto M. Gerontologia. São Paulo: Atheneu; 1997. p.403-13.

16. Marucci MFN. Avaliação das dietas oferecidas em instituições para idosos localizadas no município de São Paulo [dissertação]. São Paulo: Faculdade de Saúde Pública, Universidade de São Paulo; 1985.

17. Piloto AA, Nunes ATGL, Assis M, Frias SR. O asilo na cidade do Rio de Janeiro. Gerontologia. 1998; 6:7-12. 
18. Brasil. Conselho Federal de Nutrição. Resolução 200, de 8 de março de 1998. [Acesso em 28 maio 2004] Dispõe sobre o cumprimento das normas de definição de atribuições principal e específicas dos nutricionistas, conforme área de atuação. Diário Oficial da União [Inetrnet] 1998, Seção l; 20 de abril. Disponível em: http://www.cfn.org.br/ legislacao/resolucao/res200.htm

19. Marucci MFN. Aspectos nutricionais e hábitos alimentares de idosos, matriculados em ambulatório geriátrico [tese]. São Paulo: Faculdade de Saúde Pública, Universidade de São Paulo; 1992.

20. Aranha FQ, Barros ZF, Moura LSA, Gonçalves MCR, Barros JC, Metri JC, et al. O papel da vitamina C sobre as alterações orgânicas no idoso. Rev Nutr. 2000; 13(2):89-97.

21. Campos MTFS, Monteiro JBR, Ornelas APRC. Fatores que afetam o consumo alimentar e a nutrição do idoso. Rev Nutr. 2000; 13(3):157-65.
22. Ortega RM, Andrés $P$, Meléndez A, Turrero $E$, Gaspar M, González M, et al. Influencia de la nutrición en la capacidad funcional de un grupo de ancianos españoles. Arch Latinoam Nutr. 1992; 42(2):133-45.

23. Dirren HM. EURONUT-SENECA: a European Study of Nutrition and Health in the Elderly. Nutr Rev. 1994; 52(8):S38-S43.

24. Falque-Madrid L, Piñero-Corredor MP, Rodriguez NZ, Quintero J, Gabarrón AS, Arias-Marquez N. Estado nutricional y composición corporal de un grupo de adultos mayores no institucionalizados del Estado Zulia, Venezuela. Arch Latinoam Nutr. 1996; 46(3):190-5.

Recebido em: 23/9/2004

Versão final reapresentada em: 5/5/2005

Aprovado em: 8/6/2005 